\title{
Optical Frequency Conversion in Silica-Whispering-Gallery-Mode Microspherical Resonators
}

\author{
D. Farnesi, ${ }^{1,2}$ A. Barucci, ${ }^{1}$ G. C. Righini, ${ }^{1,2}$ S. Berneschi, ${ }^{1}$ S. Soria,,${ }^{1,}$ and G. Nunzi Conti ${ }^{1}$ \\ ${ }^{1}$ IFAC-CNR Institute of Applied Physics "N. Carrara”, Miplab, Via Madonna del Piano 10, 50019 Sesto Fiorentino (FI), Italy \\ ${ }^{2}$ Centro Studi e Ricerche "E. Fermi", P. Del Viminale 2, 00184 Rome, Italy
}

(Received 9 August 2013; published 6 March 2014)

\begin{abstract}
High quality factor whispering-gallery-mode microresonators are ideally suited for nonlinear optical interactions. We analyze, experimentally and theoretically, a variety of $\chi^{(3)}$ nonlinear interactions in silica microspheres, consisting of third harmonic generation and Raman assisted third order sum-frequency generation in the visible. A tunable, room temperature, $\mathrm{cw}$ multicolor emission in silica microspherical whispering-gallery-mode microresonators has been achieved by controlling the cavity mode dispersion and exciting nonequatorial modes for efficient frequency conversion.
\end{abstract}

DOI: 10.1103/PhysRevLett.112.093901

PACS numbers: 42.65.Ky, 42.60.Da

Whispering-gallery-mode resonators (WGMR) play an extremely important role in modern optics, being fundamental not only in many laser devices, but also as tools for very accurate frequency measurements and for nonlinear optics experiments as frequency conversion [1,2]. The extraordinary high quality factors, up to $10^{11}$, and the small volume modes make them an ideal device for shaping and enhancing light-matter interactions [3,4]. Nonlinear wave generation at low power continuous wave (cw) is still one of the biggest challenges in nonlinear optics. Whispering-gallery-mode microresonators (WGMR) can be exploited for $\mathrm{cw}$ nonlinear frequency conversion due to their properties mentioned above. However, the high circulating intensities inside the WGMR are not a sufficient condition for efficient harmonic generation, parametric and hyperparametric oscillations: it requires fulfilling phase and mode matching and energy conservation conditions [5-12].

In the case of WGMR made of silica glass, which exhibits inversion of symmetry, second order nonlinear interactions are forbidden. Here, the elemental nonlinear interaction is due to third order susceptibility $\chi^{(3)}$ effects, in which four photons are coupled. Previous work in this area, however, focuses on toroidal WGMR where most of the excitable modes are constrained to be the equatorial ones [13] or highly nonlinear materials [5,14]. Efficient generation of visible light via third order sum-frequency generation (TSFG) or four wave mixing (FWM), and third harmonic generation (THG) in silica microspheres has not been effectively explored up to now. In this Letter we demonstrate tunable optical harmonic generation with extremely narrow linewidth in silica microspheres. The

Published by the American Physical Society under the terms of the Creative Commons Attribution 3.0 License. Further distribution of this work must maintain attribution to the author(s) and the published article's title, journal citation, and DOI. selection rules for silica microspherical WGMR have been obtained systematically taking into account spatial, radial, and angular mode overlaps and material and resonator dispersion profiles. This research is aimed to develop a room-temperature $\mathrm{cw}$ miniaturized visible laser source, tunable and narrow lined to study the possibility of using optical hyperparametrical oscillations for nonclassical light generation [15] and for developing interdisciplinary applications such as lab-on-a-chip biosensors.

In the most general case of TSFG, three different waves interact with a nonlinear medium to generate a fourth wave of different frequency $\left(\omega_{\mathrm{TSFG}}=\omega_{1}+\omega_{2}+\omega_{3}\right)$. If the three input frequencies are degenerate, it will result in THG. In this case the energy conservation requires $\omega_{\mathrm{THG}}=3 \omega_{1}$. The additional phase matching condition requires $n\left(\omega_{\mathrm{THG}}\right)=$ $n\left(\omega_{p}\right)$, which in general can be fulfilled, as linear and nonlinear dispersion can be compensated for by the dense distribution of degenerate WGMs with different polar number $m$ and decreasing effective index $n_{\text {eff }}=m / k R$ [16].

In a WGMR, an additional boundary condition leads to a strict value for the resonant frequency, which may be in conflict with the strict energy conservation, meaning that $\omega_{\text {TSFG }}\left(\right.$ or $\omega_{\text {THG }}$ ) is out of resonance. The total TSFG power $P^{T}$ emitted is proportional to the overlap of the WGM eigenfunctions [Eq. (1)] [17]. These eigenfunctions are characterized by radial, azimuthal, and polar numbers, $n, l$, $m$ and they are expressed, in spherical geometry, as a product of the spherical Bessel function $j_{l}\left(k_{n} r\right)$ and spherical harmonics $Y_{l m}(\theta, \varphi)$.

$$
\begin{aligned}
P^{T} & \propto\left|\int_{0}^{R} j_{l 1}\left(k_{n l} r\right) j_{l 2}\left(k_{n 2} r\right) j_{l 3}\left(k_{n 3} r\right) j_{l}^{*}\left(k_{n} r\right) r^{2} d r\right|^{2} \\
& \times\left|\int\left[\chi^{3} Y_{l 1 m 1} Y_{l 2 m 2} Y_{l 3 m 3}\right] Y_{l m}^{*} d \Omega\right|^{2},
\end{aligned}
$$

Microspheres can be easily fabricated directly on the tip of a standard telecom fiber. In order to obtain microspheres 


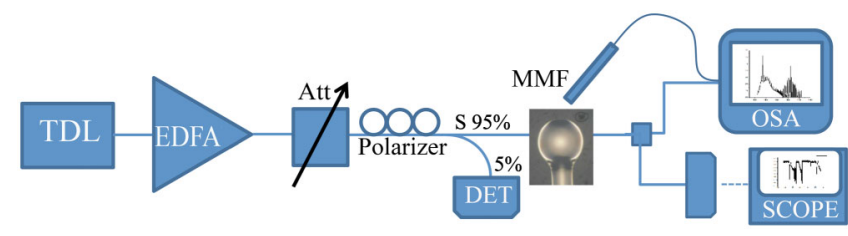

Fig. 1 (color online). Scheme of the experimental setup. TDL: Tunable diode laser; EDFA: Erbium doped fiber amplified; Att: Attenuator; S: Splitter (95:5), DET: photodiode detector; MMF: multimode fiber; OSA: Optical Spectrum Analyzer; Scope: Oscilloscope.

below the fiber diameter we melt the end of a half-tapered fiber, obtained by heating and stretching the fiber itself until it breaks. For this purpose we used a fiber fusion splicer. Using a sequence of arc discharges we fabricated spheres down to about $25 \mu \mathrm{m}$ in diameter controlling their size down to about $1 \%$ of their diameter [18]. The residual fiber stem is mounted on a translation stage with piezoelectric actuators and a positioning resolution of $20 \mathrm{~nm}$.

The experimental setup is shown in Fig. 1. The light from a tunable diode laser (TDL) is amplified with an erbium-doped fiber amplifier (EDFA) and after passing a polarization controller is coupled to the WGMR by means of a tapered fiber, also produced in-house. The laser is tuned into a resonance from high to low frequencies, which results in thermal self-locking [19] of the WGMR mode to the pump laser. THG and TSFG signals were detected on an optical spectrum analyzer (OSA) or a spectrometer by collecting with a multimode fiber (MMF, $50 \mu \mathrm{m}$ core, $0.2 \mathrm{NA}$ ), without coupling optics, the light scattered from the microsphere. Though the taper is not mode matched in the visible, a small portion of the signal is also coupled to the taper output and monitored on an OSA. A $3 \mathrm{~dB}$ splitter with one arm ending on a detector connected to an oscilloscope allows locating the resonance positions while scanning the laser.

In order to fulfill the energy conservation condition we had to select the proper sizes for the microspheres. The geometrical dispersion for the fundamental WGM can be obtained by semi-analytical calculations [20]. We included linear material dispersion and calculated the optimum size of the spherical WGMR in which the dispersion is compensated, and the frequency mismatch $\Delta \nu=3 \nu_{\mathrm{p}}-$ $\nu_{\mathrm{THG}}$ is close to zero. Indeed, Fig. 2 shows the calculated linear dispersion for THG for the fundamental WGM modes defined as $\Delta \nu_{\mathrm{WGM}}=\left(\nu_{\mathrm{WGMp}}-\nu_{\mathrm{WGMTH}} / 3\right)$, i.e., the difference between the resonance position at the pump frequency and the closest resonance around the corresponding TH frequency divided by three. The dispersion curve is calculated in the range $25-40 \mu \mathrm{m}$ for the sphere ray. We considered this range as a good compromise for keeping a high- $Q$ (around 107 : in fact the $Q$ value decreases with microsphere size because of scattering [21]) and having at the same time high power density (cavity buildup factor and mode areas both decrease with $R$, so the intensity decreases with $R^{2}$ [22]). Then, taking into account that the pump wavelength bandwidth is limited by the erbium gain band (1530-1570 nm), we obtained a set of ray ranges for which we have an optimal match $\left(\Delta \nu_{\mathrm{WGM}}=0\right)$. Figure 2 shows an oscillatory behavior of $\Delta \nu$ vs microsphere size, and therefore there are only certain ideal sphere diameters for which $\Delta \nu$ is less than the sum of their individual linewidths [17]. We focused our experiment on the range between 28 and $29 \mu \mathrm{m}$.

The inset graph in Fig. 3(a) shows a typical emission spectrum with the THG signal occurring at $519.6 \mathrm{~nm}$ for a resonant pump wavelength of $1556.95 \mathrm{~nm}$ in a microsphere

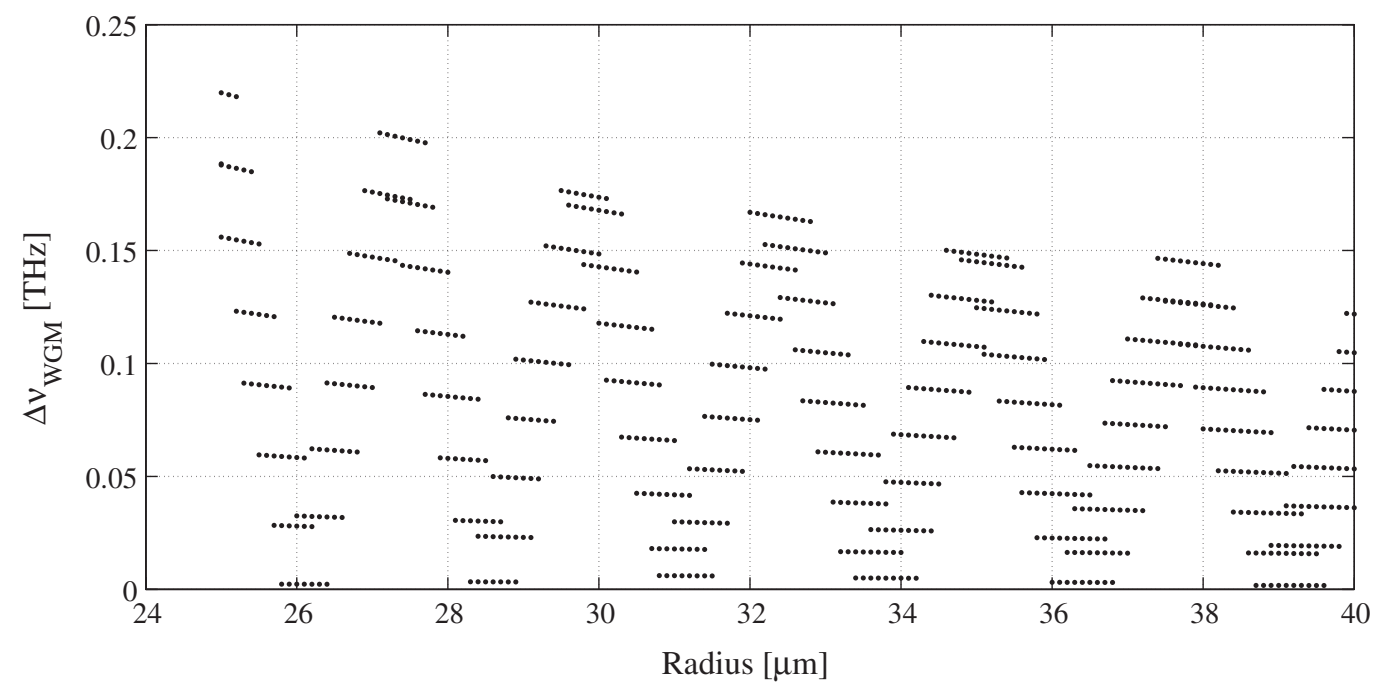

Fig. 2 Difference between the resonance position at the pump frequency (in the range of the erbium gain bandwidth) and the closest resonance around the corresponding TH frequency (divided by 3 ). The dispersion is calculated for the fundamental WGMs only and shows a set of ranges for which we have an optimal match $\left(\Delta \nu_{\mathrm{WGM}}=0\right)$. 
of $57 \pm 1 \mu \mathrm{m}$ in diameter. We collected the THG signal scattered by the WGMR with a MMF (without coupling optics) for the pump power dependence measurements. The scattered visible light shows a cubic dependence on the launched pump power, as shown in Fig. 3(a) [TH power versus $5 \%$ of the launched power measured after the splitter (see Fig. 1)]. Tuning the THG signal is possible by varying the pump wavelength in the range of the erbium band gain of the EDFA. In Fig. 3(b) we show three different TH wavelengths obtained in different microspheres by changing the pump wavelengths. The measured THG wavelengths showed a deviation comparable with the resolution of the optical spectrometer. In these cases, THG was observed without SRS (or FWM), demonstrating that under phase-matching conditions THG is enhanced in comparison with other nonlinear phenomena. In fact, TH power scales with $\left(Q_{p}\right)^{3} Q_{\mathrm{TH}} / V^{2}$, whereas other nonlinear competing phenomena scale with lower powers of $Q / V$ [23] and SRS scales with $Q_{p} Q s_{R S} / V$.

Our experiments were performed with a maximum launched pump power $\left(P_{p}\right)$ of about $80 \mathrm{~mW}$, and the
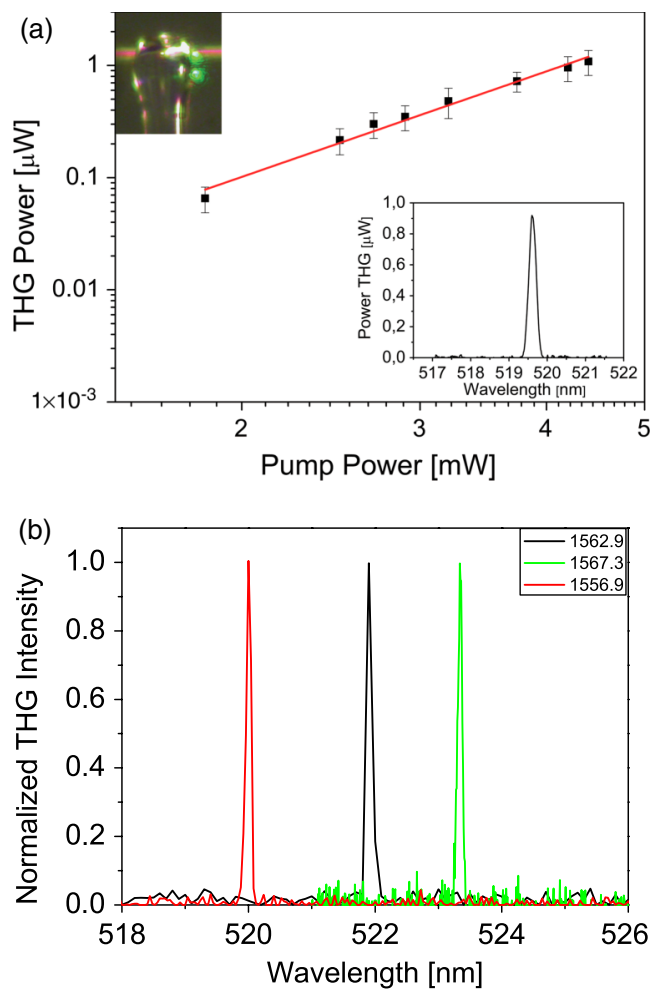

Fig. 3 (color online). (a) Measured power of the generated $\mathrm{TH}$ signal versus the $5 \%$ of the pump power, under $1556.9 \mathrm{~nm}$ pumping (log-log scale), revealing a cubic dependency as expected for THG. The red line corresponds to the linear fit (slope $3.1 \pm 0.12, R=0.99$ ). The inset graph shows the emission spectrum indicating $\mathrm{THG}$ at $519.6 \mathrm{~nm}$ when pumping at $1556.9 \mathrm{~nm}$, whereas the inset picture was taken during the spectral measurements. (b) Measured TH signal for different pump wavelengths in the erbium band gain of the EDFA. corresponding maximum THG signal $\left(P_{\mathrm{TH}}\right)$ was about $1 \mu \mathrm{W}$ for scattered [see the inset graph of Fig. 3(a)] and about $0.1 \mu \mathrm{W}$ for fiber guided THG signal (the taper is not phase matched in the visible). Thus, our maximum conversion efficiency $\eta\left(P_{\mathrm{TH}} / P_{p}\right)$ is in excess of $10^{-5}\left(10^{-6}\right.$ for fiber guided TH signal). This value represents an increase of 6 orders of magnitude over that reported in a ring resonator [24]. The inset picture of Fig. 3(a) shows a THG signal codirectional with the pump (light in the taper is coming from the left), as expected [25].

For high pump powers, other nonlinear processes are observed in the IR region, such as stimulated Raman scattering (SRS) and cascaded SRS. Lasing through SRS and cascaded SRS exhibit a clear threshold $[5,22,26]$ and a linear behavior with the pump power. In the presence of these phenomena, we have also observed TSFG in the visible, obtaining multicolor emission by tuning the pump wavelength: red, orange, yellow, and green. Figure 4 shows the measured spectra for each different color and the corresponding microscope picture of the microsphere.

In the inset pictures of Fig. 4, the TSFG signals are standing waves, since the sum involves only SRS fields. In these cases, the pump was high enough to generate several
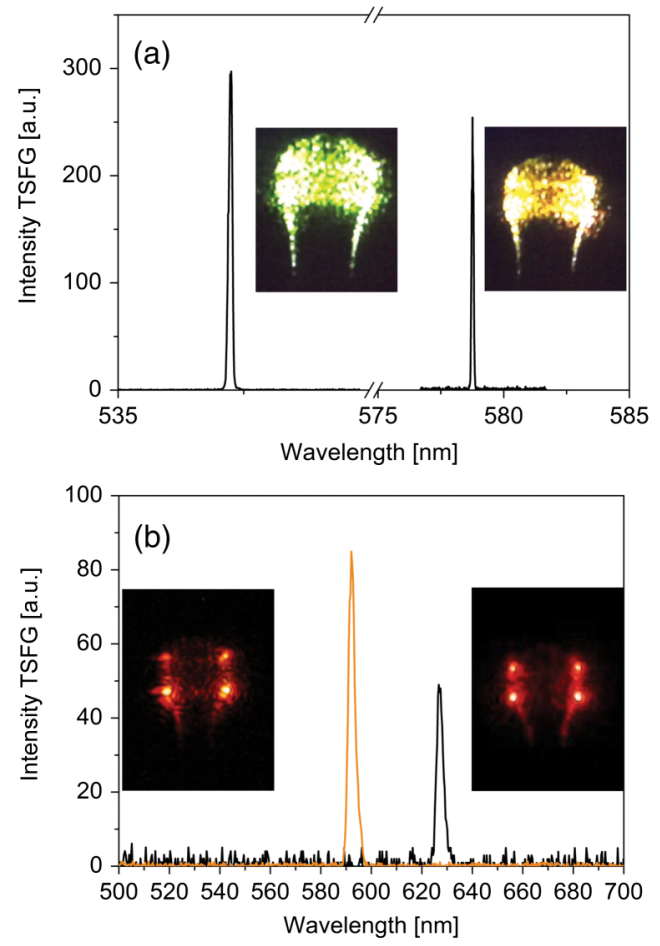

Fig. 4 (color online). Emission spectra indicating third order sum frequency generation among the pump wavelength and the cascaded Raman lines. (a) Left: at $1568.4 \mathrm{~nm}$ pump wavelength, emission at $537.24 \mathrm{~nm}$; right: at $1567 \mathrm{~nm}$ pump wavelength, with emission at $578.76 \mathrm{~nm}$; (b) Left: at $1553 \mathrm{~nm}$ pump wavelength, emitting at $592 \mathrm{~nm}$; right: at $1568.4 \mathrm{~nm}$ pump wavelength, with emission at $625 \mathrm{~nm}$. The spectra shown in (a) have been taken with an OSA, and in (b) with a handheld spectrometer. The inset pictures show standing waves. 


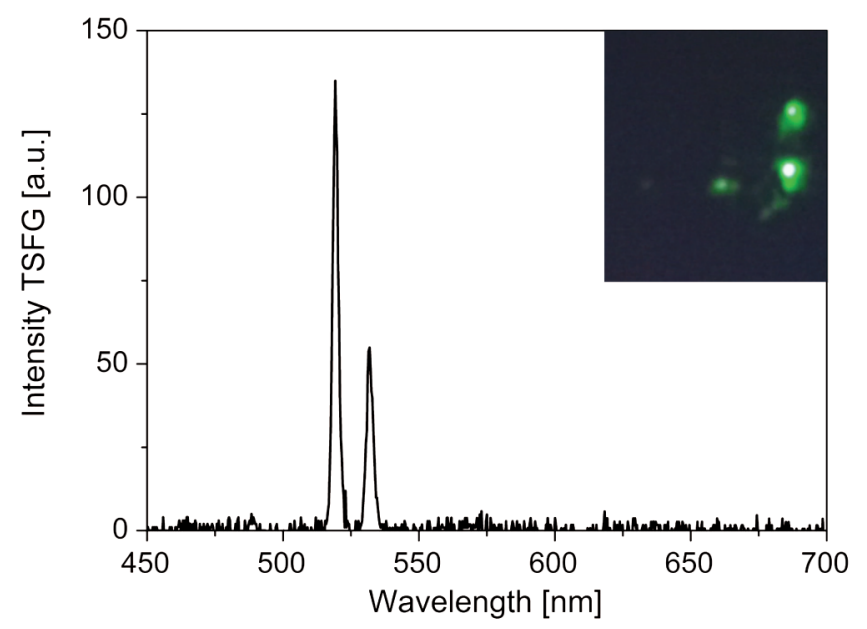

Fig. 5 (color online). Emission spectra indicating third harmonic and third order sum frequency generation among the pump wavelength at $1554.4 \mathrm{~nm}$ at $1665.4 \mathrm{~nm}$, with THG emission at $519.24 \mathrm{~nm}$ and TSFG at $531.5 \mathrm{~nm}$. The inset picture shows a traveling wave.

orders of Stokes Raman lines and Raman combs. TSFG and THG can also happen simultaneously. Figure 5 shows the spectrum with two lines, one at 519.2 and one at $531.5 \mathrm{~nm}$, the THG signal of the pump laser and the TSFG signal of the pump laser and a Raman line $\left(\omega_{\mathrm{TSFG}}=2 \omega_{p}+\omega_{\mathrm{SRS}}\right)$. In this case, the TSFG is a traveling wave, since the nonlinear polarization involves the pump laser (inset of Fig. 5). The lines are separated by $13 \mathrm{THz}$.

As can be clearly seen from all the above inset pictures, we have experimentally observed that higher order polar modes $(l-|m|>1)$ have to be excited, with the coupling taper placed far from the equatorial plane (see inset of Fig. 3) in a region corresponding to the intensity peaks of these modes [16]. These modes not only allow the phase matching conditions to be fulfilled and compensate dispersion, as previously stated, but they also provide improved mode matching which is also required for efficient TSFG/THG. We recall that TSFG power is proportional to the overlap of the WGM eigenfunctions [see Eq. (1)] and, for the degenerate case of THG, the total power is proportional to the overlap of the cubic power of the pump field with the corresponding TH field. Figure 6 shows the intensity distribution in a microsphere with a radius of $29 \mu \mathrm{m}$ for the pump mode [Fig. 6(a)] and the THG mode [Fig. 6(b)]; and the overlap [Fig. 6(c)] between the nonlinear polarization and electric field distribution of the THG for a specific example of a well matched mode. A good overlap in the regions corresponding to the fields "inversion point" is observed. The simulations of the optical modes have been done following the Mie theory with a homemade program. The pump mode is the first order mode at $1567 \mathrm{~nm}\left(n_{p}=1, l_{p}=159, m_{p}=150\right)$, and the THG mode is the first order mode at $522 \mathrm{~nm}\left(n_{\mathrm{THG}}=1\right.$, $\left.l_{\mathrm{THG}}=497, m_{\mathrm{THG}}=450\right)$. This case satisfies the quantum
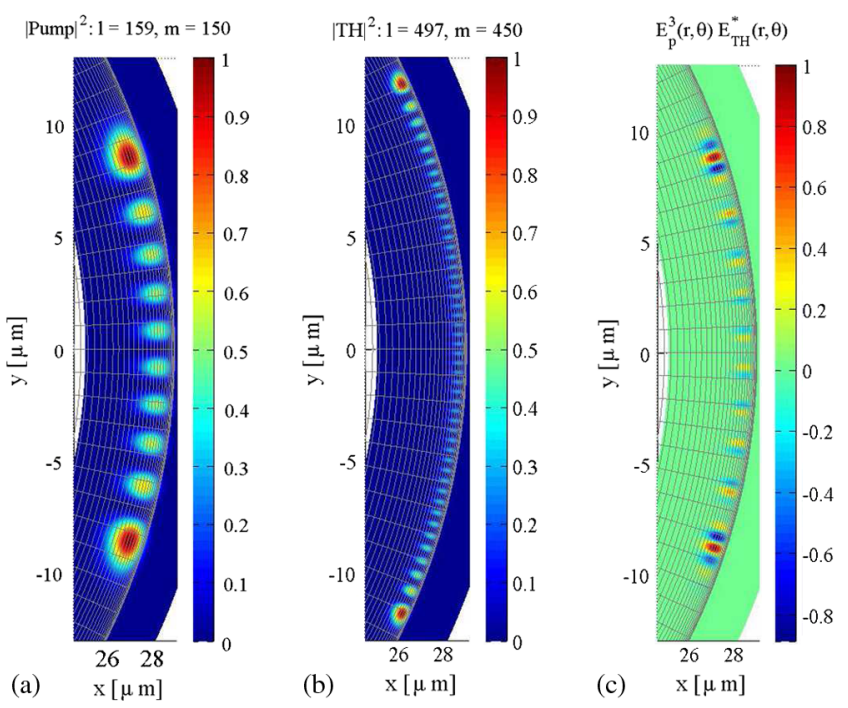

Fig. 6 (color online). (a) Intensity distribution of the pump mode, (b) intensity distribution for the THG mode, and (c) overlap of the nonlinear polarization and the electric field distribution of the THG mode. The color bar indicates the relative intensities in arbitrary units.

rules for angular momentum composition [2] and represents a perfect phase match, since the coherence length of a WGM resonance for THG is infinite for $m_{\mathrm{THG}}=$ $3 m_{p}\left(l_{\text {coh }}=\pi R /\left|m_{\mathrm{THG}}-3 m_{p}\right|\right)$, and it has the largest angular overlap $\left(l_{\mathrm{THG}} \sim 3 l_{p}\right)$ [17].

In conclusion, we observed fiber coupled THG and TSFG in silica microspherical WGMR for several pump wavelengths within the erbium band gain of the EDFA. Cavity mode dispersion was controlled by choosing suitable sizes of the silica microspheres. Phase matching was improved by exciting high order modes coincident with a particular THG and/ or TSFG frequency. In this case, there is a good spatial overlap of the resonances at the pump frequency and THG frequency. The THG and pump photons are in the states $\left|n_{\mathrm{THG}} l_{\mathrm{THG}} m_{\mathrm{THG}}\right\rangle$ and $\left|n_{p} l_{p} m_{p}\right\rangle$, respectively, and satisfied $l_{\mathrm{THG}} \sim 3 l_{p}$ and $m_{\mathrm{THG}}=3 m_{p}$. With this work, we have illustrated the great potential of WGMR as frequency converters for $\mathrm{cw}$ compact, room temperature, narrow linewidth, and tunable sources for lab-on-a-chip biosensors, spectroscopic applications, and the possibility of generation of nonclassical light.

D. Farnesi and G. C. Righini acknowledge partial funding by the "Centro Studi e Ricerche Enrico Fermi". D. Farnesi is a Material Science Ph.D. student at the University of Parma, Parma, Italy. We thank S. Pelli and C. Trono for useful discussions.

*Corresponding author. s.soria@ifac.cnr.it

[1] Y. P. Rakovich and J. F. Donegan, Laser Photonics Rev. 4, 179 (2010). 
[2] G. Kozyreff, J. L. Dominguez-Juarez, and J. Martorell, Laser Photonics Rev. 5, 737 (2011).

[3] T. J. Kippenberg, S. M. Spillane, and K. J. Vahala, Phys. Rev. Lett. 93, 083904 (2004).

[4] V.S. Ilchenko, A. A. Savchenkov, A. B. Matsko, and L. Maleki, Phys. Rev. Lett. 92, 043903 (2004).

[5] S. X. Qian and R. K. Chang, Phys. Rev. Lett. 56, 926 (1986).

[6] R. W. Boyd, Nonlinear Optics (Academic, San Diego, 2003), 2nd ed.

[7] J. U. Fürst, D. V. Strekalov, D. Elser, M. Lassen, U. L. Andersen, Ch. Marquardt, and G. Leuchs, Phys. Rev. Lett. 104, 153901 (2010).

[8] J. U. Fürst, D. V. Strekalov, D. Elser, A. Aiello, U. L. Andersen, Ch. Marquardt, and G. Leuchs, Phys. Rev. Lett. 105, 263904 (2010).

[9] T. Beckmann, H. Linnenbank, H. Steigerwald, B. Sturman, D. Haertle, K. Buse, and I. Breunig, Phys. Rev. Lett. 106, 143903 (2011).

[10] J. L. Dominguez-Juarez, G. Kozyreff, J. Martorell, Nat. Commun. 2, 254 (2011).

[11] V.S. Ilchenko, A. A. Savchenkov, A. B. Matsko, and L. Maleki, J. Opt. Soc. Am. B, 20, 1304 (2003).

[12] Y. Dumeige and P. Féron, Phys. Rev. A 74, 063804 (2006).

[13] T. Carmon and K. Vahala, Nat. Phys. 3, 430 (2007).
[14] H.-B. Lin and A. J. Campillo, Phys. Rev. Lett. 73, 2440 (1994).

[15] R. Y. Camacho, Opt. Express 20, 21977 (2012).

[16] A. Chiasera, Y. Dumeige, P. Féron, M. Ferrari, Y. Jestin, G. Nunzi Conti, S. Pelli, S. Soria, and G. C. Righini, Laser Photonics Rev. 4, 457 (2010).

[17] S. C. Hill, D. H. Leach, and R. K. Chang, J. Opt. Soc. Am. B, 10, 16 (1993).

[18] M. Brenci, R. Calzolai, F. Cosi, G. Nunzi Conti, S. Pelli, and G. C. Righini, Proc. SPIE Int. Soc. Opt. Eng., 6158, 61580S (2006).

[19] T. Carmon, L. Yang, and K. Vahala, Opt. Express 12, 4743 (2004).

[20] S. Schiller, Appl. Opt. 32, 2181 (1993).

[21] M. L. Gorodestky, A. D. Pryamikov, and V. Ilchenko, J. Opt. Soc. Am. B 17, 1051 (2000).

[22] S. M. Spillane, T. J. Kippenberg, and K. J. Vahala, Nature (London) 415, 621 (2002).

[23] L. G. Helt, M. Liscidini, and J. E. Sipe, J. Opt. Soc. Am. B 29, 2199 (2012).

[24] J. S. Levy, M. A. Foster, A. L. Gaeta, and M. Lipson, Opt. Express 19, 11415 (2011).

[25] D. H. Leach, R. K. Chang, W. P. Acker, and S. C. Hill, J. Opt. Soc. Am. B 10, 34 (1993).

[26] M. Jouravlev, D. R. Mason, and K. S. Kim, Phys. Rev. A 85, 013825 (2012). 